\title{
Caregiver stress in traumatic brain injury
}

Holly Blake, University of Nottingham.

Submitted 24 February 2008; sent back for revisions 27 March; accepted for publication following double-blind peer review 21 April 2008

\begin{abstract}
Aims: Many patients experience physical, behavioural, cognitive and emotional problems following traumatic brain injury (TBI). They may require continuing care for many years, most of which is provided by informal caregivers, such as spouses, parents, or other family members. The caregiving role is associated with a range of adverse effects including anxiety, depression, poor physical health and lowered quality of life. This article explores issues around caregiver stress; highlighting interventions for this group and areas for further research.
\end{abstract}

Methods: Literature exploring the impact of caregiving, its influencing and alleviating factors and interventions for caregivers of people with TBI is discussed, with brief critical analysis of key studies.

Findings: Research suggests that caregiver characteristics, coping strategies, their appraisal of the situation and social networks may be associated with the amount of distress experienced. Many caregivers have unmet needs such as respite care and information provision on TBI. Providing information may help to alleviate strain. Community-based family therapies providing education, support and counselling can 
help to decrease distress and improve aspects of family functioning, although evidence for these is lacking.

Conclusions: There is a need for more well-designed, controlled studies evaluating the impact of interventions to alleviate caregiver strain.

Key words: traumatic brain injury; adults; caregivers; psychosocial outcomes; social support; depression

\section{Introduction}

Traumatic brain injury (TBI) is a nondegenerative, non-congenital insult to the brain from an external mechanical force, possibly leading to permanent or temporary impairments of cognitive, physical, and psychosocial functions with an associated diminished or altered state of consciousness (Tabish et al, 2006). It is a major cause of death and disability in the western world. A review of epidemiological studies in Europe suggested an incidence of 235 hospitalized cases (including fatalities) per 100000 population (Tagliaferri et al, 2006). Advances in emergency health-care technology mean that many of those who may previously have died now survive (McAllister, 2008). People with TBI are often left with a range of temporary or permanent deficits affecting motor and cognitive function, behaviour and communication, social functioning and emotions. These impairments can have a profound effect on activities of daily living with injuries varying in severity. The 
burden of meeting patient and family needs in the years after injury is substantial (Hyder et al, 2007).

TBI causes huge upheaval to family life; once discharged from hospital, many patients require continuing care (Tennant et al, 1995) and the responsibility of care is mostly provided by family members and spouses (Knight et al, 1998). Statutory services in the UK provide acute care and a limited range of rehabilitative services. These are directed by the National Institute for Clinical Excellence on Acute Head Injuries and the National Service Framework for Long-Term Conditions (Department of Health, 2005). However, service provision is often patchy and uncoordinated (Davies et al, 2000) particularly in rural areas (Fyffe and McCubbery, 1996). Not all patients receive adequate follow-up after hospital discharge and many do not receive specialist rehabilitation treatment (Thornhill et al, 2000). Paid caregivers can play a key role in assisting people with a brain injury (McCluskey, 2000). However, informal caregivers are left to fill the gaps in service provision and this can result in physical and emotional strain for the caregivers, which may also be detrimental to patient care.

Gaining knowledge of caregivers' experiences is important in the development of supportive services for this client group. Research in this area is of mixed methodology. A narrative ('non-systematic') literature search was undertaken to explore the impact of TBI on primary caregivers and family members. Although narrative reviews are less rigorous than systematic reviews and are at risk of author bias (Critical Reviews and Advisory Group, 1996), they do provide an overview of key themes and interventions in the area. Published work from 1974-2007 was 
explored, reflecting the long-standing interest in this field. Empirical studies and review articles in CINAHL, Medline and PsychInfo were considered, with additional handsearching of journal reference lists. Search terms included 'head injury', 'traumatic brain injury', 'carers', 'caregivers', 'psychosocial', 'social support', 'strain' and 'depression'. The literature included studies published in English, conducted in the UK and abroad, involving adult patients with TBI and caregivers. The review of the literature was guided by tools from the Critical Appraisal Skills Programme (Public Health Resource Unit, 2007) and investigated the impact of caregiving, factors influencing and alleviating caregiver stress, and interventions for this group.

\section{The impact of caregiving}

Each patient with TBI experiences their injury in a unique manner and the nature of care and support required will differ between patients. As a result, the type, duration and extent of care provided by informal caregivers is not homogenous. Caregivers must adapt to living with a person who may be very different from the person he or she was before the injury. Since the early 1970s, research has highlighted the longterm impact of TBI on caregivers. Since then, a wealth of research over recent decades has increased awareness of the impact of caring for people with TBI. Informal caregivers play a key role in preventing suicide among people with moderate to severe brain injuries (Kuipers and Lancaster, 2000). It is wellestablished that caregivers are predominantly female, and there is a heavy reliance on informal networks to provide care (Chan, 2007). Although research focuses on the psychological impact, it has been recognized that caregivers' physical health may be affected (Oddy et al, 1978; Leathem et al, 1996). Furthermore, it has been suggested that caregivers' perceived physical health is lower than that of the general 
population, and that spouse's perceived health is worse than that of parents (McPherson et al, 2000).

\section{Role changes}

Caregivers often experience major changes to social roles, disturbances in their relationship with the patient, a decrease in leisure time and a reduced social circle and contact with friends. Loneliness and social isolation are frequently cited problems (Romano, 1974; Lezak, 1988). Caregivers must often assume a greater domestic workload and may take on greater responsibilities, such as looking after children, becoming a 'bread winner' or dealing with finances (McKinlay et al, 1981; Brooks and McKinley, 1983; Kreutzer et al, 1994). However, caregivers also report that the caregiving role can often prevent them from obtaining gainful employment (Chan, 2007). These changes in roles can contribute to the level of distress experienced by caregivers.

\section{Psychological problems}

Psychological problems in those caring for people following TBI are common and may include depression, anxiety, stress and burden (Oddy et al, 1978; Brook, 1991; Hall et al, 1994; Oddy 1995; Sander et al, 1997; Knight et al, 1998; Watanabe et al, 2000; Rivera et al, 2007). Although this has been well-established over the past 30 years, the generalizability of some earlier study findings is questionable; for example, Watanabe et al (2000) collected data with just nine family caregivers.

It is thought that approximately $48-60 \%$ of caregivers looking after a person with severe brain injury may experience depression (Douglas and Spellacy, 2000; Rivera et al, 2007) and feelings of strain and depression may not lessen over time (Douglas and Spellacy, 2000). In fact, Brookes et al (1986) found that caregiver strain was 
higher at 5 years post-injury than 1 year after the injury. The stress experienced by caregivers may interfere with many aspects of their lives including their ability to carry out household or work responsibilities. Living with these changes can have adverse consequences on the whole family. Standardized assessments of families of patients with TBI have revealed 'unhealthy' family functioning similar to that observed in the families of psychiatric patients (Kreutzer et al, 1994). Stress may also affect the quality of care given to patients and hinder assistance with rehabilitation activities (Kreutzer et al, 1994). In critical illness research, studies have shown that a family may communicate ineffectively with health-care professionals, distort the facts of patients' illnesses, and make decisions that are unfavourable to the patient's well-being (Twibell, 1998). The way in which the caregiver copes is therefore important since caregivers play a crucial role not only in assisting patients with activities of daily living, but also in advocacy (Jacobs, 1989). In fact, in a review of the literature, Verhaeghe et al (2005) concluded that the better the caregiver can cope, the better the patient's recovery. More recent research has also suggested that caregiver coping styles are important, with a passive coping style negatively associated with functional outcome of the person with brain injury, in terms of participation in society (Van Baalen et al, 2007). However, this study was cross-sectional in design and limited by a small sample size and missing data $(\mathrm{n}=$ 51 caregivers). In addition, the study used the Frenchay Activity Index to assess function which has been validated for use with stroke but, to the author's knowledge, has not been validated specifically for use in TBI.

\section{Positive effects and relationship to patient}

Many of the effects of brain injury on caregivers are negative. However, some caregivers report positive effects including personal reward from the caregiving 
experience, although greater personal reward is reported by parents of TBI patients than spouses (Allen et al, 1994). Similarly, partners experience more stress than parents of brain-injured individuals (Verhaeghe et al, 2005) and this may be owing to the greater role changes adapted by spouses since parents naturally accept a 'caring' role (Lezak, 1988). In a study of 180 significant others of people with TBI, Machamer et al (2002) found that the majority of caregivers reported positive aspects to caregiving, such as being happy to have had the opportunity to care for the person with $\mathrm{TBI}(93 \%)$ and feeling good about their ability as a caregiver (92\%). This study measured both positive and negative effects of caregiving on the Modified Caregiver Burden Scale (Teri et al, 1997). It should be noted, however, that the caregiving role may cause other family relationships to be neglected and siblings of people with brain injuries can also feel neglected (Wesolowski and Zencius, 1994).

\section{Factors influencing caregiver stress}

Review of the research suggests that severity of head injury bears no consistent relationship to caregiver stress. For example, some studies have shown that increased severity of brain injury is related to higher levels of caregiver stress (Livingston et al, 1985) while others have found the opposite (Oddy et al, 1978).

\section{Level of behavioural and cognitive change}

In the long-term, cognitive and behavioural problems in the brain-injured person are more likely to be associated with high levels of strain for the family than problems with physical function (Thomsen, 1984; Allen et al, 1994; Watanabe et al, 2000). Caregivers play a crucial role in implementing rehabilitation strategies to reduce cognitive impairments (such as memory training) and managing anger (McKinlay and Hickox, 1988; Campbell et al, 2007). Research has shown that the level of caregiver 
burden is associated with the degree of personality change and emotional and behavioural changes in the patient (Thomsen, 1974; Oddy et al, 1978; Lezak, 1988; Marsh et al, 1998). Such changes often include the patient's slowness, fatigability, irritability, memory disturbances, tension and anxiety, temper outbursts, depressed mood and personality changes (Brookes and McKinlay, 1983). For example, a study of 143 people by Ponsford et al (2003), using standardized measures, found that behavioural changes in a person with TBI up to 5 years after the injury are strong predictors of anxiety and depression in the care provider, although it is important to note that more than $70 \%$ of family members studied were not clinically anxious or depressed, even in situations where they were caring for their injured relative. Earlier research suggests that emotional problems in the TBI patient are the strongest predictors of caregiver burden at 1 year post-injury (Marsh et al, 1998).

\section{Caregiver beliefs}

Although it has long been established that difficult behaviours exhibited by the braininjured person can increase stress and depression in the caregiver, more recently studies have suggested that the caregiver's beliefs about these behaviours are just as important and may mediate effects of depression. Riley (2007) measured caregiver stress, depression, and social support alongside difficult behaviours in the brain injured person $(n=40)$. Results showed that more severe difficult behaviours and less social support for the caregiver were associated with increased stress and depression. Caregivers believing in their own ability to control these behaviours were less stressed. Caregivers who believed that the brain-injured person had some control over their difficult behaviours or felt that they were motivated by hostile intentions showed more signs of depression. However, there are a number of limitations to this work. First of all, although the study used standardized measures, 
these measures had not commonly been used with the population being studied. Secondly, the study was cross-sectional and therefore no assumptions can be made about causality. Thirdly, the caregivers were recruited via a voluntary brain injury organization and as a result it is not clear how representative the sample were of the population. Nevertheless, Riley's work suggests that caregiver's personal beliefs may be important and contribute to the level of stress they experience. Similarly, an earlier study of 91 family caregivers in Canada, using standardized measures of stress and coping strategies, found that the ability to reframe or positively appraise a difficult experience buffered the impact of caregiver burden on well-being (Minnes et al, 2000). However, reframing only emerged as a significant factor for certain stressrelated items on their measure, including family disharmony, lack of personal reward and terminal illness stress. Furthermore, reframing as a factor only accounted for a small proportion of the variance in overall stress scores.

\section{Cultural and gender influences}

Families identify a range of problems in the caregiving role and these appear to be similar across cultures. For example, Watanabe et al (2001) conducted interviews and questionnaire surveys with caregivers in Britain and Japan. Caregiving problems were predominantly similar although British caregivers appeared to know more about how to cope with them, and Japanese caregivers reported higher levels of social embarrassment. Although similar caregiving outcomes are observed across cultures, there may be gender differences in the way in which distress is reported (Watanabe et al, 2001). When compared with female relatives, male relatives (the majority of whom were secondary or tertiary caregivers) are likely to report their distress in terms of anger and fatigue, rather than as depression and anxiety (Perlesz et al, 2000). 


\section{Factors alleviating caregiver stress}

It seems that caregiver characteristics may play a significant role in a caregivers' ability to cope with stress. In a study of 60 caregivers completing the Centers for Epidemiologic Studies-Depression scale, Rivera et al (2007) found that caregivers of persons with TBI who report physical health problems and who exhibit ineffective problem-solving are at greater risk of depression, regardless of the time they have spent in their role as a caregiver.

\section{Coping strategies}

Patient coping strategies have been well-documented in the literature (Frank et al, 1990). Research on caregiver coping strategies has been fairly limited although this is a growing area of study (e.g. Minnes et al, 2000). Coping strategies are important since life satisfaction of the caregiver has shown to be positively affected by coping styles (Wells et al, 2005). In this study, although the sample size was larger than many published studies in this area $(n=72)$, the response rate was comparatively low and the study may have been subject to response and sample bias. Specifically, caregivers who did not respond may have been either more distressed or less so. Furthermore, the sample was recruited from a brain injury association and this group may have more motivated, i.e. actively seeking help and information, than other brain injury caregivers. Studies have identified specific coping strategies used by family members of people with brain injury, such as cognitive restructuring or reframing, maintaining enjoyable activities and pursuing emotional support through organized family support groups (Willer et al, 1991). Following a literature review, Verhaeghe et al (2005) proposed that coping occurs in stages and that there are functional and non-functional coping mechanisms. The authors further suggest that 
caregiver coping is influenced by factors such as gender, social and professional support, and the ability to have reciprocal communication or a positive emotional relationship with the patient. Readers are referred to this review for additional details of studies on caregiver coping prior to 2005.

\section{Family functioning}

Family functioning before injury appears to be a significant factor in how caregivers and relatives cope. Differences in long-term adaptation and coping efficacy are often related to pre-TBI family characteristics (Wesolowski and Zencius, 1994). Characteristics of well-functioning families include strong cohesion, understood identity, firm boundaries and open communication (Sachs, 1991). 'Vulnerable' families show less of these characteristic and can be seriously damaged by the occurrence of TBI in the family (Wesolowski and Zencius, 1994).

\section{Caregiver behavioural characteristics}

It may be that certain characteristics of the caregiver's behaviour increase their susceptibility to emotional distress. In a retrospective, cross-sectional study, Flanagan (1998) examined levels of expressed emotion in 28 caregivers of individuals with severe brain injuries. Higher levels of expressed emotion were associated with greater anxiety, although levels of expressed emotion were best predicted by caregiver status, with sole caregivers exhibiting greater expressed emotion than joint family caregivers.

\section{Social support}

Social support has been proposed as a mediator of caregiver stress and depression. In a study of 58 caregivers, Harris et al (2001) found that caregiver depression was predicted by the number of adverse effects on family members. The impact of 
adverse family effects on caregiver depression was mediated by the caregivers' perception of the effectiveness of social support. However, this study is limited in that it did not include a measure of coping, and previous research has suggested that level of coping satisfaction may be predictive of depression in this group (Knight et al, 1998). The study design was cross-sectional rather than longitudinal, therefore it spanned caregivers at several stages of the recovery process, and outcome measures were not specific to the TBI population. Evidence relating to social support is mixed, with some studies suggesting that caregivers with stronger social support ties are less likely to experience depression (Douglas and Spellacy, 2000) and others finding that social support is not associated with caregiver burden (Knight et al, 1998). Again, sample sizes in these studies were relatively small $(n=35$ and $n=$

52 respectively) limiting generalizability. A more recent study of caregiver outcomes $(n=60)$ using the Caregiver Appraisal Scale and the Social Provision Scale, found that perceived social support alone was the strongest factor in perception of caregiving 'mastery' and satisfaction with the caregiving relationship (Hanks et al, 2007). This has implications for the development of interventions for caregivers that strengthen social support networks.

\section{Interventions for caregivers}

It is known that caregiving can have profound effects on an individual and caregivers have reported specific needs, including the need for more education and resources about TBI, and social needs including more respite time for personal activities (Campbell, 1988). Although early research has identified a range of caregiver needs, studies are often limited by small sample sizes, low response rates, biased samples, non-standardized measures and descriptive approaches to study design. 


\section{Identifying caregiver needs}

One critical review (Sinnakaruppan and Williams, 2001a) focused on caregiver needs and readers are referred to this for details of individual studies. However, the key finding was that, of 13 studies identified, only six used standardized measures and the remainder used researcher-developed questionnaires. Information needs were commonly identified by caregivers, and unmet needs were often related to emotional support. Needs varied according to role relationship with the brain-injured person. In addition, it was found that unfulfilled caregiver needs could be predicted by behavioural problems in the patient. In a study of health and social needs of caregivers served by a single community rehabilitation team, Moules and Chandler (1999) reported that unmet caregiver needs were associated with poorer perceived quality of life. Despite the small sample size and the descriptive nature of the study, the findings suggest that intervention to address unmet needs in caregivers is paramount.

\section{Information provision}

Questionnaire studies and phenomenological studies have shown that caregivers often report a need for more accurate information from health professionals (Kreutzer et al, 1994; Johnson, 1995). This information is required soon after the patient is discharged from hospital and often irrespective of the severity of injury or level of functional deficit (McPherson et al, 2000). Given the poor recall and misunderstanding of information often exhibited by patients (Ley, 1990), provision of written information seems appropriate, and sometimes necessary. Research investigating the effects of providing information on levels of caregiver stress has suggested that providing information may reduce caregiver stress in acquired 
communication problems (Brumfi tt et al, 1994), dementia (Toner, 1987) and stroke (Wiles et al, 1998). In a longitudinal study of 34 caregivers of people with brain injury, Morris (2001) found that providing information booklets to caregivers could help to alleviate psychological distress. The author suggests that an information booklet for relatives of the brain-injured should become an integral part of the discharge process. This seems appropriate as although caregivers often report information needs, they do not always actively seek this information out themselves, and require prompting (McPherson et al, 2000). However, results of the Morris study must be interpreted with caution since the study lacked a control group of caregivers not receiving the intervention, and the data analysis was limited owing to small numbers of participants in comparison groups. Furthermore, caregivers in this study were not asked about previous rehabilitation involvement or psychological support which may impact on the outcome of psychological distress. In addition to early information, counselling is often required in the early stages after injury to assist with adjustment (Maitz and Sachs, 1995). Long-term assistance should focus on the transition from hospital or rehabilitation unit to home (Hosack and Rocchio, 1995).

\section{Respite}

Although informal caregivers provide the majority of continuing care in the community, there is little published research regarding the level of respite they need to continue providing quality care. However, one study found an ongoing need for respite in this group. In a survey of 85 caregivers, than (2007) found that use of respite was higher with caregivers' single marital status; increased severity of disability in the brain-injured person, high level of dependency and a greater number of days spent in a coma. This study identified caregiver's expectations of respite and factors influencing use of respite services. Although these findings emphasize the 
need for respite care, the sample were recruited from a voluntary brain injury association and so may reflect a more motivated group of caregivers who were more willing to seek support.

\section{Community care and family interventions}

Research suggests that community-based services for caregivers of people with TBI are important. Smith et al (2006) conducted an exploratory study with 17 caregivers who had received a community intervention and retrospectively compared them with 24 caregivers who had received only outpatient services. Despite design limitations, the findings suggested that those receiving community services were more likely to have their needs met, less likely to have 'dysfunctional' families, and less likely to feel emotionally burdened. However, the effect of these services needs to be explored further in a prospective controlled study using standardized measures of outcome. Family interventions include elements of assessment, educational workshops, follow-up workshops, individualized family support and specialist marital counselling (Tyerman and Booth, 2001). Perlesz and O'Loughlan (1998) monitored the outcomes of 15 families (32 individuals) seeking family counselling in a publiclyfunded family therapy centre over a 2-year period: before commencing counselling; 12 months after the commencement of counselling; and 24 months after the commencement of counselling. Caregiver strain was reduced and levels of distress decreased for both patient and caregiver. Family conflict decreased and there were improvements to family cohesion and adjustment across the study period. However, improvements to marital adjustment and anger were not maintained across time and by the end of the study had returned to the pre-counselling level. A review specifically of family interventions after brain injury and other chronic conditions accessed 31 journal articles (using set inclusion and exclusion criteria), only four of 
which were in brain injury (Boschen et al, 2007). The key finding from this review was the lack of methodological rigour in the evidence-base and, although it spanned a range of chronic conditions, this finding is applicable to research on caregivers and TBI. The authors concluded that there is currently no strong research evidence supporting any specific intervention method for family caregivers of individuals with TBI or any of the other chronic condition groups surveyed. The authors referred to an 'abundance of anecdotal, descriptive, and quasi-experimental support' which exists in the rehabilitation literature. A review of articles relating to community support systems for caregivers of people with brain injury was conducted by Sinnakaruppan and Williams (2001b) who identified only seven articles at that time. These included professionally-led behavioural and cognitive interventions to assist caregivers to adjust to problem behaviours in the brain-injured person. The authors found that only one of the studies used standardized outcome measures, the remainder evaluated the interventions using self-report items or subjective interviews. They found that samples were often biased and lacked control groups. This review identified a need for larger, controlled studies using standardized measures as outcomes to evaluate caregiver intervention programmes.

\section{Specific patient rehabilitation programmes}

Some studies have looked at the impact of specific patient rehabilitation programmes on the caregiver. Bowen et al (2001) implemented a new neuropsychological rehabilitation service and compared three groups, one receiving the new service at an early stage, one receiving it later and one receiving only standard care $(n=96)$. The authors examined caregiver emotional distress and how informed caregivers felt about TBI and available resources, and compared outcomes between groups. Results showed less caregiver strain in the two groups in which the brain-injured 
individuals had received the new rehabilitation service. Fraas et al (2007) reported the outcomes of a community- based programme for meeting the long-term needs of survivors of TBI. Quantitative and qualitative evaluation showed that the programme met many needs of people with TBI, although social support for caregivers was an unmet need. However, the focus group in this study included only one caregiver.

\section{DISCUSSION}

In addition to rehabilitating the person with $\mathrm{TBI}$, therapists play a key role in easing caregivers' burden by identifying those caregivers in need of further support. The therapist is well-placed to provide medical information about TBI and its consequences, and provide training in the management of physical, cognitive and emotional impairments. Therapists can engage in discussion of role and relationship changes, and provide strategies to improve communication. They can also provide training in stress management techniques and help caregivers to access local and national community resources and support groups. Therefore it is important for therapists and health professionals to be aware of the issues and research around caregiver stress, so that they can assist the patient and their family appropriately. Caregiving in TBI is associated with a range of adverse consequences for caregiver health, psychological well-being and quality of life. There are also indirect effects on the quality of patient care. The majority of published research focuses on the negative outcomes of caregiving and families who are unable to cope. However, despite the trauma of the initial injury, it should be recognized that many families go on to cope very well and it has been suggested that more research should be conducted with caregivers who have adjusted well to the TBI (Perlesz et al, 2000). Although the caregiver's experience can be associated with many negative factors individual to their caregiving situation, the majority of relatives do report overall 
positive experiences. Nevertheless, caregiving exerts considerable stress and a high proportion of caregivers experience strain. This strain may be mediated by many factors, including previous family functioning, caregiver appraisal of the situation and level of social support.

\section{Limitations}

This article does not systematically review the literature but presents some of the key concepts and published evidence in the field. Although much of the literature published over past years highlights important associations between caregiver stress and predicting variables, services and support strategies have changed over recent years and so less recent evaluations of interventions to alleviate strain may not reflect current service improvements and developments. Many of the published studies are limited by small sample sizes, single centre approaches and a lack of standardized outcome measures. Studies often have descriptive methodologies and monitor a single group over time, or they are based on retrospective methods of data collection.

Some studies are hampered by sample bias or response bias. There appears to be few studies of samples with diverse ethnic backgrounds, focusing on caregiving relationships other than marital partners or parents (such as unmarried but cohabiting domestic partnerships), children, or focusing on the extent of access to services. Furthermore, findings of studies are difficult to compare owing to differences in design and methodologies. It seems that the literature is lacking in rigorous, controlled studies, conducted prospectively over longer periods of time and measuring outcome using standardized assessment tools. These methodological limitations of research relating to TBI caregivers have been identified by previous 
reviews (Chwalisz, 1992; Kreutzer et al, 1992; DeJong, 1999) although more recent research evidence is often subject to similar methodological limitations. Also, this population is heavily researched and studies often compete for participants. Therefore, it can be difficult to access large numbers of participants and also to identify a suitable comparison group in brain injury. Furthermore, a large proportion of studies that have identifi ed factors associated with alleviating strain in caregivers of people with TBI have not given rise to corresponding interventions that have been well-evaluated. Service developers therefore need to be mindful of evidence in the development of interventions for brain injury caregivers.

\section{CONCLUSIONS}

The fact that caregiving for persons with TBI can be a stressful role is undisputed. Supporting caregivers is therefore an essential part of long-term care following TBI. Research has identified factors associated with caregiver distress and differences between predictors of stress for particular caregiving groups, such as spouses or parents. There are many interventions which may help to alleviate stress including the provision of information, support groups and self-help resources, family support and counselling, caregiver training and respite care. However, the variation in design and methodology in the published literature makes comparison between studies and generalization of findings difficult. The literature is lacking in large-scale controlled trials to evaluate the outcome of interventions for caregivers of people with TBI. In order to target services, more well-designed research is needed that evaluates the impact of interventions to reduce stress in caregivers of brain-injured individuals.

Informal caregivers provide long-term support for individuals with traumatic brain injury (TBI). 


\section{Key Points}

- Caregiver strain is prevalent in this group and increased by unmet needs.

- Strain can be moderated by coping styles, personal appraisal and social support.

- Interventions include information provision, training, support groups, respite and counselling. Therapists are well-placed to identify caregivers who need assistance and, where appropriate, provide these interventions.

- Well-designed large-scale controlled trials are required to evaluate the impact of interventions on caregiver strain in TBI.

Conflicts of interest: None. 


\section{References}

Allen K, Linn R, Gutierrez H (1994) Family burden following traumatic brain injury. Rehabil Psychol 39: 29-48

Boschen K, Gargaro J, Gan C, Gerber G, Brandys C (2007) Family interventions after acquired brain injury and other chronic conditions: a critical appraisal of the quality of the evidence. NeuroRehabilitation 22(1): 19-41

Bowen A, Tennant A, Neumann V, Chamberlain M (2001) Neuropsychological rehabilitation for traumatic brain injury: do carers benefi t? Brain Inj 15(1): 29-38

Brooks D (1991) The head injured family. J Clin Exp Neuropsychol 13: 155-88

Brooks N, Campsie L, Symington C, Beattie A, McKinlay W (1986) The five year outcome of severe blunt head injury: a relative's view. J Neurol Neurosurg Psychiatry 49: $764-70$

Brooks N, McKinlay W (1983) Personality and behavioural change after severe blunt head injury - A relative's view. J Neurol Neurosurg Psychiatry 46: 336-44

Brumfi tt S, Atkinson J, Greated C (1994) The carer's response to written information about acquired communication problems. Aphasiology 8: 583-90

Campbell C (1988) Needs of relatives and helpfulness of support groups in severe head injury. Rehabil Nurs 13: 320-5

Campbell L, Wilson F, McCann J, Kernahan G, Rodgers R (2007) Single case experimental design study of carer facilitated errorless learning in a patient with severe memory impairment following TBI. NeuroRehabilitation 22:325-33 
Chwalisz K (1992) Perceived stress and caregiver burden after brain injury: A theoretical integration. Rehabil Psychol 37: 189-203

Chan J (2007) Carers' perspective on respite for persons with acquired brain injury. Int J Rehabil Res 30(2): 137-46

Critical Reviews and Advisory Group (1996) Introduction to Systematic reviews. School for Health and Related Research (ScHARR), University of Sheffield, UK Davies C, Hawley C, Stilwell J, Stilwell P (2000). Views of service changes in UK brain injury rehabilitation. British Journal of Therapy and Rehabilitation 7: 521-4

DeJong G, Batavia A, Williams J (1990) Who is responsible for the life long wellbeing of a person with a head injury? J Head Trauma Rehabil 5: 9-22

DeJong G (1999) Access to brain injury rehabilitation and other post-acute services. In: Ragnarsson K, ed. Report of the NIH consensus development process on the rehabilitation of persons with traumatic brain injury. United States Department of Health and Human Services, Bethesda, MD: 121-4

Department of Health (2005) National Service Framework for Long-Term Conditions: Older People and Disability Policy Planning Unit, DH, London

Douglas J, Spellacy F (2000) Correlates of depression in adults with severe traumatic brain injury and their carers. Brain Inj 14(1): 71-88

Flanagan D (1998) A retrospective analysis of expressed emotion (EE) and affective distress in a sample of relatives caring for traumatically brain-injured (TBI) family members. Br J Clin Psychol 37 (Part 4): 431-9

Frank R, Haut A, Smick M (1990) Coping and family functions after closed head injury. Brain Inj 4: 289-95 
Fraas M, Balz M, Degrauw W (2007) Meeting the long-term needs of adults with acquired brain injury through community- based programming. Brain Inj 21(12): $1267-81$

Fyffe C, McCubbery J (1996) 'If only we had somewhere to turn ...' the service needs of people with acquired brain injury in a rural community. Aust $\mathrm{J}$ Rural Health 4(4): $232-6$

Hall K, Karzmark P, Stevens M (1994) Family stressors in traumatic brain injury: a two year follow up. Arch Phys Med Rehabil 75: 876-84

Hanks RA, Rapport LJ, Vangel S (2007) Caregiving appraisal after traumatic brain injury: The effects of functional status, coping style, social support and family functioning. NeuroRehabilitation 22 (1): 43-52

Harris J, Godfrey H, Partridge F, Knight R (2001) Caregiver depression following traumatic brain injury (TBI): a consequence of adverse effects on family members? Brain Inj 15 (3): 223-38

Hosack K, Rocchio C (1995) Serving families of persons with severe brain injury in an era of managed care. J Head Trauma Rehabil 10(2): 57-65

Hyder AA, Wunderlich CA, Puvanachandra P, Gururaj G, Kobusingye OC (2007) The impact of traumatic brain injuries: a global perspective. NeuroRehabilitation 22(5): 341-53

Jacobs H (1988) The Los Angeles head injury survey: procedures and initial findings. Arch Phys Med Rehabil 69:425-31

Jacobs H (1989) Long-term family intervention. In: Ellis D, Christensen A, eds. Neuropsychological treatment after brain injury. Kluwer Academic, Boston: 297-316 
Johnson B (1995) One family's experience with head injury: A phenomenological study. Journal of Neuroscience Nursing 27(2): 113-18

Knight R, Devereux R, Godfrey H (1998) Caring for a family member with a traumatic brain injury. Brain Inj 12: 467-81

Kreutzer JS, Marwitz JH, Kepler K (1992) Traumatic Brain Injury: family response and outcome. Arch Phys Med Rehabil 73(8): 771-8

Kreutzer J, Gervasio A, Camplair P (1994) Primary caregivers' psychological status and family functioning after traumatic brain injury. Brain Inj 8: 197-210

Kuipers P, Lancaster A (2000) Developing a suicide prevention strategy based on the perspectives of people with brain injuries. J Head Trauma Rehabil 15(6): 127584

Leathem J, Heath E, Wolley C (1996) Relatives' perceptions of role change, social support and stress after traumatic brain injury. Brain Inj 10: 27-38

Ley P (1990) Communicating with patients: Improving communication satisfaction and compliance. Chapman and Hall, London

Lezak M (1988) Brain damage is a family affair. J Clin Exp Neuropsychol 10: 111-23

Livingston M, Brooks D, Bond M (1985) Three months after severe head injury: psychiatric and social impact on relatives. J Neurol Neurosurg Psychiatry 48: 870-5

Machamer J, Temkin N, Dikmen S (2002) Significant other burden and factors related to it in traumatic brain injury. J Clin Exp Neuropsychol 24(4): 420-33

Maitz E, Sachs P (1995) Treating families of individuals with traumatic brain injury from a family systems perspective. J Head Trauma Rehabil 10(2): 1-11 
Marsh N, Kersel D, Havill J, Sleigh J (1998) Caregiver burden at 6 months following severe traumatic brain injury. Brain Inj 12: 225-38

McAllister TW (2008) Neurobehavioural sequelae of traumatic brain injury:

evaluation and management. World Psychiatry 7(1): 3-10

McCluskey A (2000) Paid attendant carers hold important and unexpected roles which contribute to the lives of people with brain injury. Brain Inj 14(11): 943-57

McKinlay W, Brooks D, Bond M (1981) The short term outcome of severe blunt head injury as reported by relatives of the injured person. J Neurol Neurosurg Psychiatry 44: $527-33$

McKinlay W, Hickox A (1988) How can families help in the rehabilitation of the head injured? J Head Trauma Rehabil 3(4): 64-72

McPherson K, McNaughton H, Pentland B (2000) Information needs of families when one member has a severe brain injury. Int J Rehabil Res 23(4): 295-301

McPherson K, Pentland B, McNaughton H (2000) Brain injury-the perceived health of carers. Disabil Rehabil 22(15): 683-9

Minnes P, Graffi S, Nolte M, Carlson P, Harrick L (2000) Coping and stress in Canadian family caregivers of persons with traumatic brain injury. Brain Inj 14: 73748

Morris K (2001) Psychological distress in carers of head injured individuals: the provision of written information. Brain Inj 15(3): 239-54

Moules S, Chandler B (1999) A study of the health and social needs of carers of traumatically brain-injured individuals served by one community rehabilitation team. Brain Inj 13(12): 983-93 
Oddy M (1995) He's no longer the same person: how families adjust to personality change after head injury. In: Chamberlain M, Neumann V, Tennant A, eds. Traumatic Brain Injury Rehabilitation: Services, Treatments and Outcomes. Chapman and Hall Medical, London: 167-179

Oddy M, Humphrey M, Uttley D (1978) Stresses upon the relatives of head-injured patients. Br J Psychiatry 133: 507-13

Perlesz A, Kinsella G, Crowe S (2000) Psychological distress and family satisfaction following traumatic brain injury: injured individuals and their primary, secondary, and tertiary carers. J Head Trauma Rehabil 15(3): 909-29

Perlesz A, O'Loughlan M (1998) Changes in stress and burden in families seeking therapy following traumatic brain injury: a follow-up study. Int J Rehabil Res 21(4): $339-54$

Ponsford J, Olver J, Ponsford M, Nelms R (2003) Long-term adjustment of families following traumatic brain injury where comprehensive rehabilitation has been provided. Brain Inj 17: 453-68

Public Health Resource Unit (2007) Critical Appraisal Skills Programme. http://www.phru.nhs.uk/Pages/PHD/CASP.htm (accessed 24 April 2008)

Riley G (2007) Stress and depression in family carers following traumatic brain injury: the influence of beliefs about difficult behaviours. Clin Rehabil 21(1): 82-8 Rivera P, Elliott TR, Berry JW, Grant JS, Oswald K (2007) Predictors of caregiver depression among community- residing families living with traumatic brain injury. NeuroRehabilitation 22(1): 3-8 
Romano M (1974) Family responses to traumatic head injury. Scand J Rehabil Med 6: $1-4$

Sachs PR (1991) Treating families of brain-injury survivors. Springer, New York Sander A, High W, Hannay H (1997) Predictors of psychological health in caregivers of patients with closed head injury. Brain Inj 11: 235-49

Sinnakaruppan I, Williams D (2001a) Family carers and the adult head-injured: a critical review of carers' needs. Brain Inj 15(8): 653-72

Sinnakaruppan I, Williams D (2001b) Head injury and family carers: a critical appraisal of case management programmes in the community. Int J Rehabil Res 24(1): 35-42

Smith M, Vaughan F, Cox L et al (2006) The impact of community rehabilitation for acquired brain injury on carer burden: an exploratory study. J Head Trauma Rehabil 21(1): 78-81

Tabish A, Lone NA, Afzal WM, Salam A (2006) The incidence and severity of injury in children hospitalised for traumatic brain injury in Kashmir. Injury 37(5): 410-15 Tagliaferri F, Compagnone C, Korsic M, Servadei F, Kraus J (2006) A systematic review of brain injury epidemiology in Europe. Acta Neurochir (Wien) 148: 255-68 Tennant A, Macdermott N, Neary D (1995) The long-term outcome of head injury: implications for service planning. Brain Inj 9(6): 595-605

Teri L, Logsdon RG, Uomoto J, McCurry SM (1997) Behavioural treatment of depression in dementia patients: A controlled clinical trial. J Gerontol B Psychol Sci Soc Sci 52(4): P159-66 
Thomsen I (1974) The patient with severe head injury and his family. Scand J Rehabil Med 6: 180-3

Thomsen I (1984) Late outcome of very severe blunt head trauma: a 10-15 year second follow-up. J Neurol Neurosurg Psychiatry 47: 260-8

Thornhill S, Teasdale G, Murray G, McEwen J, Roy C, Penny K (2000) Disability in young people and adults one year after head injury: a prospective cohort study. BMJ 320: $1631-5$

Toner H (1987) Effectiveness of a written guide for carers of dementia sufferers. British Journal of Clinical and Social Psychiatry 5: 24-6

Twibell R (1998) Family coping during critical illness. Dimens Crit Care Nurs 17: $100-12$

Tyerman A, Booth J (2001) Family interventions after traumatic brain injury: a service example. NeuroRehabilitation 16(1): 59-66

Van Baalen B, Ribbers GM, Medema-Meulepas D, Pas MS, Odding E, Stam HJ (2007) Being restricted in participation after a traumatic brain injury is negatively associated by passive coping style of the caregiver. Brain Inj 21(9): 925-31 Verhaeghe S, Defl oor T, Grypdonck M (2005) Stress and coping among families of patients with traumatic brain injury: a review of the literature. J Clin Nurs 14(8): $1004-12$

Watanabe Y, Shiel A, Asami T, Taki K, Tabuchi K (2000) An evaluation of neurobehavioural problems as perceived by family members and levels of family stress 1-3 years following traumatic brain injury in Japan. Clin Rehabil 14: 172-7 
Watanabe Y, Shiel A, McLellan D, Kurihara M, Hayashi K (2001) The impact of traumatic brain injury on family members living with patients: a preliminary study in Japan and the UK. Disabil Rehabil 23(9): 370-8

Wells R, Dywan J, Dumas J (2005) Life satisfaction and distress in family caregivers as related to specific behavioural changes after traumatic brain injury. Brain Inj 19(13):1105-15

Wesolowski M, Zencius A (1994) A practical guide to head injury rehabilitation: A focus on postacute residential treatment. Plenum Press, New York

Wiles R, Pain H, Buckland S (1998) Providing appropriate information to patients and carers following a stroke. J Adv Nurs 28: 794-801

Willer BS, Allen KM, Liss M, Zicht MS (1991) Problems and coping strategies of individuals with traumatic brain injury and their spouses. Arch Phys Med Rehabil 72(7): 460-4 